Bulletin of the Section of Logic

Volume 46:1/2 (2017), pp. 135-149

http://dx.doi.org/10.18778/0138-0680.46.1.2.10

Andrzej Indrzejczak

\title{
CUT ELIMINATION THEOREM FOR NON-COMMUTATIVE HYPERSEQUENT CALCULUS
}

\begin{abstract}
Hypersequent calculi (HC) can formalize various non-classical logics. In [9] we presented a non-commutative variant of $\mathrm{HC}$ for the weakest temporal logic of linear frames Kt4.3 and some its extensions for dense and serial flow of time. The system was proved to be cut-free $\mathrm{HC}$ formalization of respective temporal logics by means of Schütte/Hintikka-style semantical argument using models built from saturated hypersequents. In this paper we present a variant of this calculus for Kt4.3 with a constructive syntactical proof of cut elimination.
\end{abstract}

Keywords: temporal logic, linear time, hypersequent calculus, cut elimination.

\section{Introduction}

Hypersequent calculus (HC) is one of the generalizations of ordinary sequent calculus well crafted for dealing with certain classes of non-classical logics. Since its discovery by G. Pottinger [17] and A. Avron [1], a lot of cutfree formalizations of many logics, including modal, many-valued, relevant, paraconsistent and fuzzy logics was proposed. Important examples of its application may be found in: Avron [2,3] Baaz, Ciabattoni and Fermüller [4], Ciabattoni, Galatos and Terui [6], Metcalfe, Olivetti and Gabbay [14], Indrzejczak [7, 8], Kurokawa [13], Lahav [10], Bednarska, and Indrzejczak [5], Lellmann [11] and [12].

The main feature of this kind of formal system is the application of finite collections of sequents (hypersequents) as the basic items for which 
rules of the system are defined. Usually such collections are interpreted as sets or multisets and the order of sequents does not matter. However, if we define hypersequents as finite lists of sequents we may extend our expressive resources. Such kind of hypersequent calculus was introduced in Indrzejczak [9] for dealing with the minimal temporal logic of linear frames HCKt4.3 and all its extensions covering features of density and past or future seriality. Application of non-commutative hypersequents nicely corresponds to linear order of time points. Suitable rules allow for syntactic modelling of both forward and backward transmission of data on the time axis.

A calculus provided in [9] is cut-free but its adequacy is proved by means of semantical techniques. In particular, the completeness is demonstrated by means of Schütte/Hintikka's style argument showing how to construct a countermodel for any unprovable hypersequent. This is nice solution and allows for well-behaved root-first proof search. However a syntactical proof of cut elimination would be also welcome. The lack of commutativity of sequents makes the solution of this problem harder than in set/multiset-based hypersequents but fortunately, not impossible. In this paper we provide a fully syntactical, constructive proof of cut elimination for a slightly modified variant of the $\mathrm{HC}$ in [9]. The proof is based on the strategy applied by Schütte [18] for the one-sided version of sequent calculus.

\section{2. $\quad$ Temporal logic Kt4.3}

We will consider the weakest temporal logics of linear time Kt4.3 in standard bimodal language with countable set of propositional variables $V A R$, ordinary boolean constants and two Priorean unary temporal operators: $\square^{F}$ (always in the future) and $\square^{P}$ (always in the past). Dual operators $\diamond^{F}$ and $\diamond^{P}$ are treated as definitional shortcuts. One can axiomatize Kt4.3 by the addition to any Hilbert system for classical propositional logic the following schemata:

$$
\begin{aligned}
& \mathrm{KF} \square^{F}(\varphi \rightarrow \psi) \rightarrow\left(\square^{F} \varphi \rightarrow \square^{F} \psi\right) \\
& \mathrm{KP} \square^{P}(\varphi \rightarrow \psi) \rightarrow\left(\square^{P} \varphi \rightarrow \square^{P} \psi\right) \\
& \mathrm{PF} \varphi \rightarrow \square^{P} \diamond^{F} \varphi \\
& \operatorname{FP} \varphi \rightarrow \square^{F} \diamond^{P} \varphi \\
& \quad 4 \square^{F} \varphi \rightarrow \square^{F} \square^{F} \varphi
\end{aligned}
$$




\section{$\mathrm{LF} \square^{P} \varphi \wedge \square^{F} \varphi \wedge \varphi \rightarrow \square^{F} \square^{P} \varphi$ $\mathrm{LP} \square^{P} \varphi \wedge \square^{F} \varphi \wedge \varphi \rightarrow \square^{P} \square^{F} \varphi$}

The system is closed under MP (modus ponens) and two rules of necessitation:

$$
\begin{aligned}
& \vdash \varphi / \vdash \square^{F} \varphi \\
& \vdash \varphi / \vdash \square^{P} \varphi
\end{aligned}
$$

Kt4.3 is adequate with respect to the class of relational frames $\langle T, R\rangle$ where acessibility relation $R$ on the nonempty set of time points $T$ is transitive and satisfies the conditions of future and past linearity (or connectedness):

$$
\begin{aligned}
& \forall t, t^{\prime}, t^{\prime \prime}\left(R t t^{\prime} \wedge R t t^{\prime \prime} \rightarrow R t^{\prime} t^{\prime \prime} \vee R t^{\prime \prime} t^{\prime} \vee t^{\prime}=t^{\prime \prime}\right) \\
& \forall t, t^{\prime}, t^{\prime \prime}\left(R t^{\prime} t \wedge R t^{\prime \prime} t \rightarrow R t^{\prime} t^{\prime \prime} \vee R t^{\prime \prime} t^{\prime} \vee t^{\prime}=t^{\prime \prime}\right)
\end{aligned}
$$

It may be shown that Kt4.3 is also characterised by the narrower class of linear structures, where instead of these two conditions it holds the condition of trichotomy:

$$
\forall t, t^{\prime}\left(R t t^{\prime} \vee R t^{\prime} t \vee t=t^{\prime}\right)
$$

Models $\mathfrak{M}$ are built on frames by the addition of valuation function $v: V A R \longrightarrow \mathcal{P}(T)$. Formulae are evaluated at the points of the model in the standard way by means of recursively defined satisfaction relation $\vDash$. In particular, the clauses for temporal operators are the following:

$\mathfrak{M}, t \vDash \square^{F} \varphi$ iff $\mathfrak{M}, t^{\prime} \vDash \varphi$ for every $t^{\prime}$ such that $R t t^{\prime}$

$\mathfrak{M}, t \vDash \square^{P} \varphi$ iff $\mathfrak{M}, t^{\prime} \vDash \varphi$ for every $t^{\prime}$ such that $R t^{\prime} t$

$\mathfrak{M}, t \vDash \diamond^{F} \varphi$ iff $\mathfrak{M}, t^{\prime} \vDash \varphi$ for some $t^{\prime}$ such that $R t t^{\prime}$

$\mathfrak{M}, t \vDash \diamond^{P} \varphi$ iff $\mathfrak{M}, t^{\prime} \vDash \varphi$ for some $t^{\prime}$ such that $R t^{\prime} t$

A formula $\varphi$ is $\mathbf{K t 4 . 3}$-valid $(\models \varphi)$ iff it is satisfied at every point of every Kt4.3-Model. A formula is falsifiable if it is nonvalid, i.e. $\mathfrak{M}, t \not \models \varphi$ for some $\mathfrak{M}$ and $t$.

\section{Hypersequent calculus}

Hypersequent calculus for Kt4.3 defined below is a slightly changed variant of the system in [9]. We define hypersequents as finite lists of Gentzen's sequents and apply the following notation: 
- $\Gamma \Rightarrow \Delta$ or $s$ (usually with subscripts) for sequents; note that $\Gamma, \Delta$ are finite, possibly empty, sets of formulae.

- $\wedge \Gamma(\vee \Gamma)$ for the conjunction (disjunction) of all elements of $\Gamma$.

- $s_{1}|\cdots| s_{n}, G, H, G_{i}, H_{i}$ stand for hypersequents; in particular $G, G_{i}$ will always denote nonempty hypersequent, whereas $H, H_{i}$ will be used if we admit that it is empty.

- $H_{1}|s| H_{2}$ (or $H_{1}|\Gamma \Rightarrow \Delta| H_{2}$ ) stand for hypersequents with displayed sequent $s$ (or $\Gamma \Rightarrow \Delta$ ).

The calculus for $\mathbf{K t 4 . 3}$ consists of the following rules:

$$
\begin{aligned}
& \text { (AX) } H_{1}|p, \Gamma \Rightarrow \Delta, p| H_{2} \\
& \text { (Cut) } \frac{H_{1}|\Gamma \Rightarrow \Delta, \varphi| H_{2} \quad H_{1}|\varphi, \Gamma \Rightarrow \Delta| H_{2}}{H_{1}|\Gamma \Rightarrow \Delta| H_{2}} \\
& (\neg \Rightarrow) \frac{H_{1}|\Gamma \Rightarrow \Delta, \varphi| H_{2}}{H_{1}|\neg \varphi, \Gamma \Rightarrow \Delta| H_{2}} \\
& (\Rightarrow \neg) \frac{H_{1}|\varphi, \Gamma \Rightarrow \Delta| H_{2}}{H_{1}|\Gamma \Rightarrow \Delta, \neg \varphi| H_{2}} \\
& (\wedge \Rightarrow) \frac{H_{1}|\varphi, \psi, \Gamma \Rightarrow \Delta| H_{2}}{H_{1}|\varphi \wedge \psi, \Gamma \Rightarrow \Delta| H_{2}} \\
& (\Rightarrow \wedge) \frac{H_{1}|\Gamma \Rightarrow \Delta, \varphi| H_{2} \quad H_{1}|\Gamma \Rightarrow \Delta, \psi| H_{2}}{H_{1}|\Gamma \Rightarrow \Delta, \varphi \wedge \psi| H_{2}} \\
& (\Rightarrow \vee) \frac{H_{1}|\Gamma \Rightarrow \Delta, \varphi, \psi| H_{2}}{H_{1}|\Gamma \Rightarrow \Delta, \varphi \vee \psi| H_{2}} \\
& (\vee \Rightarrow) \frac{H_{1}|\varphi, \Gamma \Rightarrow \Delta| H_{2} \quad H_{1}|\psi, \Gamma \Rightarrow \Delta| H_{2}}{H_{1}|\varphi \vee \psi, \Gamma \Rightarrow \Delta| H_{2}} \\
& (\Rightarrow \rightarrow) \frac{H_{1}|\varphi, \Gamma \Rightarrow \Delta, \psi| H_{2}}{H_{1}|\Gamma \Rightarrow \Delta, \varphi \rightarrow \psi| H_{2}} \\
& (\rightarrow \Rightarrow) \frac{H_{1}|\Gamma \Rightarrow \Delta, \varphi| H_{2} \quad H_{1}|\psi, \Gamma \Rightarrow \Delta| H_{2}}{H_{1}|\varphi \rightarrow \psi, \Gamma \Rightarrow \Delta| H_{2}} \\
& \left(\Rightarrow \square^{F}\right) \frac{H|\Gamma \Rightarrow \Delta| \Rightarrow \varphi}{H \mid \Gamma \Rightarrow \Delta, \square^{F} \varphi} \\
& \left(\square^{F} \Rightarrow\right) \frac{H_{1}|\Gamma \Rightarrow \Delta| \square^{F} \varphi, \varphi, \Pi \Rightarrow \Sigma \mid H_{2}}{H_{1}\left|\square^{F} \varphi, \Gamma \Rightarrow \Delta\right| \Pi \Rightarrow \Sigma \mid H_{2}} \\
& \left(\Rightarrow \square^{P}\right) \frac{\Rightarrow \varphi|\Gamma \Rightarrow \Delta| H}{\Gamma \Rightarrow \Delta, \square^{P} \varphi \mid H} \\
& \left(\square^{P} \Rightarrow\right) \frac{H_{1}\left|\square^{P} \varphi, \varphi, \Gamma \Rightarrow \Delta\right| \Pi \Rightarrow \Sigma \mid H_{2}}{H_{1}|\Gamma \Rightarrow \Delta| \square^{P} \varphi, \Pi \Rightarrow \Sigma \mid H_{2}} \\
& \left(\Rightarrow \square^{F^{\prime}}\right) \\
& \frac{|\Gamma \Rightarrow \Delta| \Rightarrow \varphi|\Pi \Rightarrow \Sigma| \quad|\Gamma \Rightarrow \Delta| \Pi \Rightarrow \Sigma, \varphi|\quad| \Gamma \Rightarrow \Delta\left|\Pi \Rightarrow \Sigma, \square^{F} \varphi\right|}{H_{1}\left|\Gamma \Rightarrow \Delta, \square^{F} \varphi\right| \Pi \Rightarrow \Sigma \mid H_{2}} \\
& \left(\Rightarrow \square^{P^{\prime}}\right) \\
& \frac{|\Gamma \Rightarrow \Delta| \Rightarrow \varphi|\Pi \Rightarrow \Sigma| \quad|\Gamma \Rightarrow \Delta, \varphi| \Pi \Rightarrow \Sigma|\quad| \Gamma \Rightarrow \Delta, \square^{P} \varphi|\Pi \Rightarrow \Sigma|}{H_{1}|\Gamma \Rightarrow \Delta| \Pi \Rightarrow \Sigma, \square^{P} \varphi \mid H_{2}}
\end{aligned}
$$

In schemata of $\left(\Rightarrow \square^{F^{\prime}}\right)$ and $\left(\Rightarrow \square^{P^{\prime}}\right)$ we omitted parameters $H_{1}, H_{2}$ in all premisses for better readability. 
In [9] we provided also a detailed analysis of the rules and rationale behind their shape so now we only briefly point out two important things:

1. All rules satisfy the subformula property.

2. Hypersequents defined as finite lists of sequents allow for easy representation of time flow. Informally, every sequent corresponds to some point on the time axis and if $H_{1}\left|s_{i}\right| \cdots\left|s_{j}\right| H_{2}$, then the time point represented by $s_{i}$ is earlier than that represented by $s_{j}$.

The proof of a hypersequent $G$ is defined in the usual way as a tree of hypersequents with $G$ as the root, axioms as leaves and all other nodes regulated by the application of rules. The height of a proof of a hypersequent is defined as the number of nodes in the longest branch of this proof.

Example proof of the instance of $L F$ looks like that.

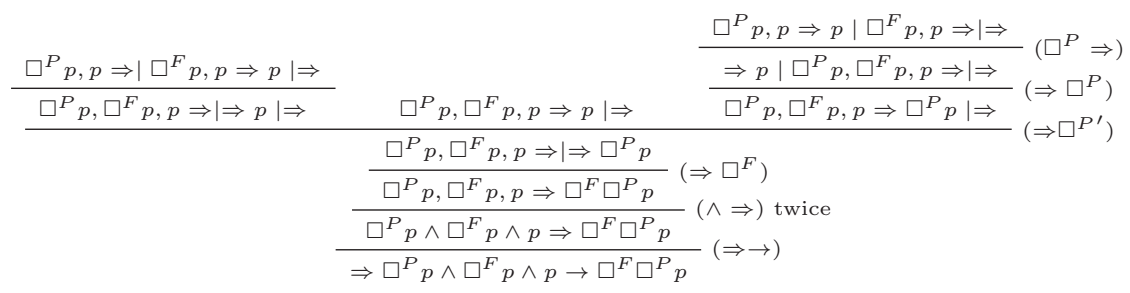

All these rules are certainly sufficient for cut-free deductions of other axioms, as the reader may easily check.

In what follows we will denote with $\vdash G$ the provability of $G$ in our HC for Kt4.3.. For brevity we will simply state all the results without constant mentioning the system.

As we noted the present system is but a small variant of the one presented in [9]. The differences between the two systems are the following:

1. Sequents are formed from finite sets in the present system;

2. Axioms are restricted to atomic formulae as active formulae, whereas in [9] this restriction is absent;

3. (Cut) is primitive in the present version;

4. $\left(\square^{F} \Rightarrow\right)$ and $\left(\square^{P} \Rightarrow\right)$ are slightly different in both systems.

The first difference is due to the fact that we apply Schütte strategy of proof which works better for sets. Restriction to atomic formulae is needed 
for a proof of invertibility of rules. Finally, the new shape of two temporal rules is essential for the proof of cut elimination to go through. Respective rules in [9] were non-local in the sense that the side formula $\varphi$ could be placed in any other sequent in the premiss on the right (in $\left(\square^{F} \Rightarrow\right)$ ) or on the left (in $\left(\square^{P} \Rightarrow\right)$ ) of the active sequent. The present rules are local in the sense that $\varphi$ occurs in the immediate neighbour-sequent on the right or on the left and with the principal formula repeated. This change makes possible step by step control of inferences applied in the proof.

\section{Soundness}

We must recall the principles of interpretation of non-commutative hypersequents after [9].

Satisfaction of a sequent at a state in a model is defined in a standard way:

$\mathfrak{M}, t \vDash \Gamma \Rightarrow \Delta$ iff $\mathfrak{M}, t \vDash \wedge \Gamma \rightarrow \vee \Delta$,

and

$\mathfrak{M}, t \not \models \Gamma \Rightarrow \Delta$ iff $\mathfrak{M}, t \not \models \wedge \Gamma \rightarrow \vee \Delta$,

We extend semantical notions to non-commutative hypersequents in the following way:

Definition 1. For any Kt4.3-model $\mathfrak{M}$ and hypersequent $G=s_{1}|\cdots| s_{n}$ :

- $\mathfrak{M} \models G$ iff for all states $t_{1}, \ldots, t_{n}$ of $\mathfrak{M}$ : if $t_{1} R t_{2} R \ldots R t_{n}$, then for some $i \leq n, \mathfrak{M}, t_{i} \vDash s_{i}$;

- $\models G$ ( $G$ is Kt4.3-valid) iff $\mathfrak{M} \models G$ for every $\mathfrak{M}$.

Note that in consequence: $\not \models G$ iff there is $\mathfrak{M}$ such that $\mathfrak{M} \not \forall G$ and this means that there are $t_{1}, \ldots, t_{n}$ such that $t_{1} R t_{2} R \ldots R t_{n}$ and $t_{1} \not \models s_{1}, \ldots$, $t_{n} \not \models s_{n}$.

One can easily prove:

Lemma 1 (Validity-preservation). All rules are validity-preserving

We will show by contraposition two cases: $\left(\square^{F} \Rightarrow\right)$ and $\left(\Rightarrow \square^{F^{\prime}}\right)$. 
$\left(\square^{F} \Rightarrow\right)$. Assume that $\not \forall H_{1}\left|\square^{F} \varphi, \Gamma \Rightarrow \Delta\right| \Pi \Rightarrow \Sigma \mid H_{2}$. Hence there is a model $\mathfrak{M}$ such that $\mathfrak{M}, t \not \models \square^{F} \varphi, \Gamma \Rightarrow \Delta$ and $\mathfrak{M}, t^{\prime} \not \models \Pi \Rightarrow \Sigma$, for some $t, t^{\prime}$ such that $R t t^{\prime}$. In particular, $\mathfrak{M}, t \vDash \square^{F} \varphi$, hence $\mathfrak{M}, t^{\prime} \vDash \varphi$ and, by transitivity also $\mathfrak{M}, t^{\prime} \vDash \square^{F} \varphi$, which means that this model falsifies $H_{1}|\Gamma \Rightarrow \Delta| \square^{F} \varphi, \varphi, \Pi \Rightarrow \Sigma \mid H_{2}$.

$\left(\Rightarrow \square^{F^{\prime}}\right)$. Assume that $\not \models H_{1}\left|\Gamma \Rightarrow \Delta, \square^{F} \varphi\right| \Pi \Rightarrow \Sigma \mid H_{2}$. Hence there is a model $\mathfrak{M}$ such that $\mathfrak{M}, t \not \models \Gamma \Rightarrow \Delta, \square^{F} \varphi$ and $\mathfrak{M}, t^{\prime} \not \models \Pi \Rightarrow \Sigma$, for some $t, t^{\prime}$ such that $R t t^{\prime}$. In particular, $\mathfrak{M}, t \not \models \square^{F} \varphi$ which means that there is some $t^{\prime \prime}$ such that $R t t^{\prime \prime}$ and $\mathfrak{M}, t^{\prime \prime} \not \models \varphi$. Future linearity implies $R t^{\prime} t^{\prime \prime} \vee R t^{\prime \prime} t^{\prime} \vee t^{\prime}=t^{\prime \prime}$. If we take the first case, then $\mathfrak{M}, t^{\prime} \not \models \square^{F} \varphi$ and the third premiss, i.e. $H_{1}|\Gamma \Rightarrow \Delta| \Pi \Rightarrow \Sigma, \square^{F} \varphi \mid H_{2}$ is falsified by this model. If we take the second choice, then we can assign $t^{\prime \prime}$ to $\Rightarrow \varphi$. This way we obtain an augmented model with $t^{\prime \prime}$ inserted between $t$ and $t^{\prime}$ which falsifies the first premiss, i.e. $H_{1}|\Gamma \Rightarrow \Delta| \Rightarrow \varphi|\Pi \Rightarrow \Sigma| H_{2}$. The last choice falsifies the second premiss. i.e. $H_{1}|\Gamma \Rightarrow \Delta| \Pi \Rightarrow \Sigma, \varphi \mid H_{2}$. Hence if the conclusion is falsifiable, then at least one of the premisses must be falsifiable.

As a consequence we obtain

Theorem 1 (Soundness). If $\vdash G$, then $\models G$

\section{Preliminary Results}

Before proving completeness and cut elimination theorem of the system we must show some auxiliary results. In particular, we must show that most of the rules are invertible and for that we also need to provide proofs of admissibility of some structural rules. In this respect our proof is somewhat similar to the proof of Poggiolesi [15] for the HC system for S5 which is an adaptation of Dragalin's strategy of proving cut admissibility to HC setting. In fact our proof is even more dependent on invertibility and in this respect it is rather an adaptation of Schütte's strategy [18] of proof to $\mathrm{HC}$ setting.

Let us consider the following structural rules:

$$
(I W) \frac{H_{1}|\Gamma \Rightarrow \Delta| H_{2}}{H_{1}\left|\Gamma^{\prime} \Rightarrow \Delta^{\prime}\right| H_{2}} \quad(E W) \frac{H_{1} \mid H_{2}}{H_{1}|s| H_{2}}
$$


where in $(I W)$ (internal weakening) $\Gamma \subseteq \Gamma^{\prime}, \Delta \subseteq \Delta^{\prime}$ (at least one strictly subsumed) and in $(E W)$ (external weakening) at least one of $H_{1}, H_{2}$ is nonempty.

In the proofs below we detail only the cases for $\square^{F}$ leaving "mirror" proofs for $\square^{P}$ to the reader. Also we will use an abbreviation $(I C)$ for both (internal) contraction rules.

The following results hold:

Lemma 2. $\quad$ 1. $\vdash H_{1}|\varphi, \Gamma \Rightarrow \Delta, \varphi| H_{2}$;

2. $(I W)$ is height-preserving admissible;

3. $(E W)$ is admissible.

Proof: ad. 1: By induction on the complexity of $\varphi$. In case of boolean formulas it is trivial. For $\square^{F} \varphi$ we need subsidiary induction on the number of sequents to the right of the active sequent (in case of $\square^{P} \varphi$ we perform induction on the number of sequents to the left). The basis is simple:

$$
\frac{G|\Gamma \Rightarrow \Delta| \square^{F} \varphi, \varphi \Rightarrow \varphi}{\frac{G\left|\square^{F} \varphi, \Gamma \Rightarrow \Delta\right| \Rightarrow \varphi}{G \mid \square^{F} \varphi, \Gamma \Rightarrow \Delta, \square^{F} \varphi}\left(\square^{\mathrm{F}} \Rightarrow\right)}\left(\Rightarrow \square^{\mathrm{F}}\right)
$$

where the top-hypersequent is derivable by the induction hypothesis. For the induction step consider the application of $\left(\Rightarrow \square^{F^{\prime}}\right)$ with the conclusion:

$$
G\left|\square^{F} \varphi, \Gamma \Rightarrow \Delta, \square^{F} \varphi\right| \Theta \Rightarrow \Lambda \mid H
$$

and the following premisses:

$$
\begin{aligned}
& G\left|\square^{F} \varphi, \Gamma \Rightarrow \Delta,\right| \Rightarrow \varphi|\Theta \Rightarrow \Lambda| H \\
& G\left|\square^{F} \varphi, \Gamma \Rightarrow \Delta\right| \Theta \Rightarrow \Lambda, \varphi \mid H
\end{aligned}
$$

and

$$
G\left|\square^{F} \varphi, \Gamma \Rightarrow \Delta\right| \Theta \Rightarrow \Lambda, \square^{F} \varphi \mid H
$$

To each of the premisses we may apply $\left(\square^{\mathrm{F}} \Rightarrow\right)$ in the following way:

$$
\frac{G|\Gamma \Rightarrow \Delta,| \square^{F} \varphi, \varphi \Rightarrow \varphi|\Theta \Rightarrow \Lambda| H}{G\left|\square^{F} \varphi, \Gamma \Rightarrow \Delta,\right| \Rightarrow \varphi|\Theta \Rightarrow \Lambda| H}\left(\square^{\mathrm{F}} \Rightarrow\right)
$$




$$
\frac{G|\Gamma \Rightarrow \Delta| \square^{F} \varphi, \varphi, \Theta \Rightarrow \Lambda, \varphi \mid H}{G\left|\square^{F} \varphi, \Gamma \Rightarrow \Delta\right| \Theta \Rightarrow \Lambda, \varphi \mid H}\left(\square^{\mathrm{F}} \Rightarrow\right)
$$

and

$$
\frac{G|\Gamma \Rightarrow \Delta| \square^{F} \varphi, \varphi \cdot \Theta \Rightarrow \Lambda, \square^{F} \varphi \mid H}{G\left|\square^{F} \varphi, \Gamma \Rightarrow \Delta\right| \Theta \Rightarrow \Lambda, \square^{F} \varphi \mid H}\left(\square^{\mathrm{F}} \Rightarrow\right)
$$

In the first and the second case the starting hypersequent is provable by the induction hypothesis of the main induction. As for the third, note that the number of the sequents to the right was decreased in the premiss so this hypersequent is also derivable by the induction hypothesis of the subsidiary induction.

ad. 2. This is trivial since all rules, including temporal and the additional structural ones, are context insensitive and the addition of new formulae does not harm their correctness.

ad. 3. The proof is by induction on the height of the proof of $H_{1} \mid H_{2}$. The problem is only with the temporal rules since all of them assume dependence of active sequents on their neighbours. There are three cases for $\square^{F} \varphi$. However in [9] we provided a proof of admissibility of $(E W)$ in the presence of $\left(\Rightarrow \square^{F}\right)$ and $\left(\Rightarrow \square^{F^{\prime}}\right)$ and these rules are the same in the present system. So we must consider only the case of $\left(\square^{F} \Rightarrow\right)$ which is different. In fact, the only problematic situation is when we want to add a new sequent immediately to the right of the active sequent. Let the left part of the conclusion $\left(H_{1}\right.$ in the schema of $\left.(E W)\right)$ be of the form $G \mid \square^{F} \varphi, \Gamma \Rightarrow \Delta$, the right part $\left(H_{2}\right)$ be of the form $\Theta \Rightarrow \Lambda \mid H$ and a new sequent placed between the two be $\Pi \Rightarrow \Sigma$. So we have a proof ending with:

$$
\frac{G|\Gamma \Rightarrow \Delta| \square^{F} \varphi, \varphi, \Theta \Rightarrow \Lambda \mid H}{G\left|\square^{F} \varphi, \Gamma \Rightarrow \Delta\right| \Theta \Rightarrow \Lambda \mid H}\left(\square^{\mathrm{F}} \Rightarrow\right)
$$

and we obtain the result as follows:

$$
\frac{G|\Gamma \Rightarrow \Delta| \varphi, \Pi \Rightarrow \Sigma\left|\square^{F} \varphi, \varphi, \Theta \Rightarrow \Lambda\right| H}{\frac{G|\Gamma \Rightarrow \Delta| \square^{F} \varphi, \varphi, \Pi \Rightarrow \Sigma|\Theta \Rightarrow \Lambda| H}{G\left|\square^{F} \varphi, \Gamma \Rightarrow \Delta\right| \Pi \Rightarrow \Sigma|\Theta \Rightarrow \Lambda| H}\left(\square^{\mathrm{F}} \Rightarrow\right)}
$$

The first line is by the induction hypothesis $(\Pi \Rightarrow \Sigma$ inserted $)$ and $(I W)$ ( $\varphi$ added to its antecedent). This example (as well as cases of $\left(\Rightarrow \square^{F}\right)$ 
and $\left(\Rightarrow \square^{F^{\prime}}\right)$ ) shows why $(E W)$ is not height-preserving admissible; the additional application of $\left(\square^{\mathrm{F}} \Rightarrow\right)$ increases the height of this proof by 1 .

Thanks to the point 1 . of the lemma we are able to prove all axioms. Cut allows for simulation of MP and EW for simulation of necessitation rules. Hence, by the completeness of Hilbert calculi for these logics, we are in a position to conclude with:

Theorem 2 (Completeness). If $\models G$, then $\vdash G$.

Before we proceed with cut elimination we must prove height-preserving invertibility of almost all logical rules.

Lemma 3. 1. All boolean rules are height-preserving invertible.

2. The rightmost and the central premiss of the rules $\left(\Rightarrow \square^{F^{\prime}}\right)$ and $\left(\Rightarrow \square^{P^{\prime}}\right)$ are height-preserving invertible.

Proof: Consider the case of $\left(\Rightarrow \square^{F^{\prime}}\right)$ and its rightmost premiss. We show that if $\vdash G\left|\Gamma \Rightarrow \Delta, \square^{F} \varphi\right| \Theta \Rightarrow \Lambda \mid H$ then $\vdash G|\Gamma \Rightarrow \Delta| \Theta \Rightarrow \Lambda, \square^{F} \varphi \mid$ $H$ by induction on the height of the proof of the premiss. The basis is trivial. For induction step we must consider the cases of all rules as leading to its proof. If $\square^{F} \varphi$ is principal, then the rightmost premiss of ( $\Rightarrow \square^{F^{\prime}}$ ) provides already a proof (of lower height) of the required hypersequent. If it is non-principal we consider the premiss(es) of suitable rules, apply the induction hypothesis to them and then a respective rule. We will illustrate the point with $\left(\Rightarrow \square^{F^{\prime}}\right.$ ) but applied to some other formula $\square^{F} \psi$ (again with $G$ and $H$ omitted for readability in the premisses). We have:

$\frac{\left|\Gamma \Rightarrow \Delta, \square^{F} \varphi\right| \Rightarrow \psi|\Theta \Rightarrow \Lambda| \quad\left|\Gamma \Rightarrow \Delta, \square^{F} \varphi\right| \Theta \Rightarrow \Lambda, \psi|\quad| \Gamma \Rightarrow \Delta, \square^{F} \varphi\left|\Theta \Rightarrow \Lambda, \square^{F} \psi\right|}{G\left|\Gamma \Rightarrow \Delta, \square^{F} \varphi, \square^{F} \psi\right| \Theta \Rightarrow \Lambda \mid H}$

which is transformed into:

$\frac{|\Gamma \Rightarrow \Delta| \Rightarrow \psi\left|\Theta \Rightarrow \Lambda, \square^{F} \varphi\right||\Gamma \Rightarrow \Delta| \Theta \Rightarrow \Lambda, \square^{F} \varphi, \psi|| \Gamma \Rightarrow \Delta, \square^{F} \varphi\left|\Theta \Rightarrow \Lambda, \square^{F} \varphi, \square^{F} \psi\right|}{G\left|\Gamma \Rightarrow \Delta, \square^{F} \psi\right| \Theta \Rightarrow \Lambda, \square^{F} \varphi \mid H}$

REMARK: In fact all rules are invertible except $\left(\square^{F} \Rightarrow\right)$ and $\left(\square^{P} \Rightarrow\right)$. Additive cut is trivially height-preserving invertible by $(I W)$. Also $\left(\Rightarrow \square^{F}\right)$ and $\left(\Rightarrow \square^{P}\right)$ are invertible as well as $\left(\Rightarrow \square^{F^{\prime}}\right)$ and $\left(\Rightarrow \square^{P^{\prime}}\right)$ with respect to the 
leftmost premiss, but in these cases we may only demonstrate it semantically which is of no help in our cut elimination proof. Fortunately, for this aim we do not need their invertibility.

\section{Cut Elimination}

We are in the position to provide our central result:

Theorem 3. Cut is eliminable.

Proof: We prove eliminability of cut by double induction: on the complexity of cut formula, and on the height of a proof of the right premiss. It is sufficient to consider a proof ending in an uppermost application of cut.

In the basis of the main induction (on the complexity of cut formula) we prove for any atomic formula $p$ that cut with it as cut formula is eliminable. The proof goes by subsidiary induction on the height of the right premiss.

The basis is trivial: the right premiss is an axiom and, if $p$ is not its active formula, then it is enough to delete $p$. If $p$ is active, then the conclusion follows from the left premiss.

In the induction step we assume that cut is eliminable for any proof of the right premiss with the height lower than $n$. Let the proof be of the height $n$, we must check all rules applicable in the last step.

As an example consider the case of $(\vee \Rightarrow)$ as the last rule:

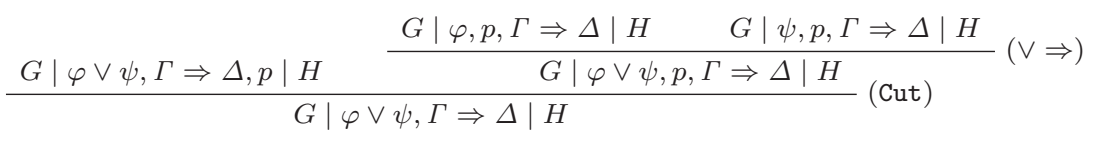

is replaced with

$$
\frac{G|\varphi, \Gamma \Rightarrow \Delta, p| H \quad G|p, \varphi, \Gamma \Rightarrow \Delta| H}{(\vee \Rightarrow) \frac{G|\varphi, \Gamma, \Rightarrow \Delta| H}{G|\varphi \vee \psi, \Gamma, \Rightarrow \Delta| H}} \quad \frac{G|\psi, \Gamma \Rightarrow \Delta, p| H \quad G|p, \psi, \Gamma \Rightarrow \Delta| H}{G|\psi, \Gamma, \Rightarrow \Delta| H}
$$

with both cuts of lower height. Note that since we eliminate additive cut we must apply invertibility lemma twice to the left premiss of the old cut to unify premisses of new cuts. Note also that we considered the case with the position of $p$ in the active sequent, but the cases where $p$ is placed in any other sequent occurring in $G$ or $H$ lead to exactly the same transformations. This remark applies also to further cases, where - for the sake of readability 
- we always put the cut formula in some of the active sequents but in case it occurs in some other sequent makes no significant changes in the proof.

The most complicated case is that of $\left(\Rightarrow \square^{\mathrm{F}^{\prime}}\right)$. We have the following instance of $(C u t)$ :

$$
\frac{G\left|\Gamma \Rightarrow \Delta, \square^{F} \varphi, p\right| \Theta \Rightarrow \Lambda|H \quad G| p, \Gamma \Rightarrow \Delta, \square^{F} \varphi|\Theta \Rightarrow \Lambda| H}{G\left|\Gamma \Rightarrow \Delta, \square^{F} \varphi\right| \Theta \Rightarrow \Lambda \mid H} \text { (Cut) }
$$

where the right premiss was deduced by $\left(\Rightarrow \square^{F^{\prime}}\right.$ ) in the following way:

$$
\frac{G|p, \Gamma \Rightarrow \Delta| \Rightarrow \varphi|\Theta \Rightarrow \Lambda| H \quad G|p, \Gamma \Rightarrow \Delta| \Theta \Rightarrow \Lambda, \varphi|H \quad G| p, \Gamma \Rightarrow \Delta\left|\Theta \Rightarrow \Lambda, \square^{F} \varphi\right| H}{G\left|p, \Gamma \Rightarrow \Delta, \square^{F} \varphi\right| \Theta \Rightarrow \Lambda \mid H}
$$

Again the main trouble is that when we shift cuts upward we must unify premisses. The original left premiss of cut cannot be simply applied thrice to premisses of the respective application of $\left(\Rightarrow \square^{\mathrm{F}^{\prime}}\right)$ since they differ not only in the position of cut formula but in one case even with the number of sequents. We apply twice inversion lemma to the left premiss of $(C u t)$ and make two cuts of lower height with two premisses of $\left(\Rightarrow \square^{\mathrm{F}^{\prime}}\right.$ ) which introduced the right premiss of (Cut):

$$
\frac{G|\Gamma \Rightarrow \Delta, p| \Theta \Rightarrow \Lambda, \varphi|H \quad G| p, \Gamma \Rightarrow \Delta|\Theta \Rightarrow \Lambda, \varphi| H}{G|\Gamma \Rightarrow \Delta| \Theta \Rightarrow \Lambda, \varphi \mid H} \text { (Cut) }
$$

and

$$
\frac{G|\Gamma \Rightarrow \Delta, p| \Theta \Rightarrow \Lambda, \square^{F} \varphi|H \quad G| p, \Gamma \Rightarrow \Delta\left|\Theta \Rightarrow \Lambda, \square^{F} \varphi\right| H}{G|\Gamma \Rightarrow \Delta| \Theta \Rightarrow \Lambda, \square^{F} \varphi \mid H} \text { (Cut) }
$$

Unfortunately we cannot apply inversion lemma to the left premiss of cut to obtain the sequent which agree with the leftmost premiss of suitable instance of $\left(\Rightarrow \square^{\mathrm{F}^{\prime}}\right)$. Instead we may apply $(E W)$ to the left premiss of the original application of cut, and $(I W)$ to the leftmost premiss of $\left(\Rightarrow \square^{\mathrm{F}^{\prime}}\right)$ leading originally to the right premiss of cut. We obtain:

$$
\frac{G\left|\Gamma \Rightarrow \Delta, \square^{F} \varphi, p\right| \Rightarrow \varphi|\Theta \Rightarrow \Lambda| H \quad G\left|p, \Gamma \Rightarrow \Delta, \square^{F} \varphi\right| \Rightarrow \varphi|\Theta \Rightarrow \Lambda| H}{G\left|\Gamma \Rightarrow \Delta, \square^{F} \varphi\right| \Rightarrow \varphi|\Theta \Rightarrow \Lambda| H} \text { (Cut) }
$$

Fortunately, this cut is also eliminable by the induction hypothesis since $(I W)$ is height-preserving admissible and the induction is only on the 
height of the right premiss. In order to combine these three hypersequents in a new application of $\left(\Rightarrow \square^{\mathrm{F}^{\prime}}\right)$ we must add by $(I W)$ also $\square^{F} \varphi$ to $\Delta$. Finally we obtain the result:

$$
\frac{\left|\Gamma \Rightarrow \Delta, \square^{F} \varphi\right| \Rightarrow \varphi|\Theta \Rightarrow \Lambda| \quad\left|\Gamma \Rightarrow \Delta, \square^{F} \varphi\right| \Theta \Rightarrow \Lambda, \varphi|\quad| \Gamma \Rightarrow \Delta, \square^{F} \varphi\left|\Theta \Rightarrow \Lambda, \square^{F} \varphi\right| H}{G\left|\Gamma \Rightarrow \Delta, \square^{F} \varphi\right| \Theta \Rightarrow \Lambda \mid H}
$$

Note that in contrast to transformations on the right premiss obtained by boolean rule this time we must additionaly use implicit internal contraction. The same applies to cases where the right premiss was deduced by the application of $\left(\Rightarrow \square^{\mathrm{F}}\right)$ and $\left(\square^{\mathrm{F}} \Rightarrow\right)$.

For inductive step of the main induction we must consider all possible compound cut-formulae. In case of boolean formulae it is sufficient to apply inversion lemma. Let us illustrate the point with conjunction. Applying inversion lemma to both premisses of cut we get: $G|\Gamma \Rightarrow \Delta, \psi| H, G \mid$ $\Gamma \Rightarrow \Delta, \varphi \mid H$ and $G|\varphi, \psi, \Gamma \Rightarrow \Delta| H$. With the help of $(I W)$ we obtain:

$$
\frac{G|\Gamma \Rightarrow \Delta, \psi| H \quad \frac{G|\psi, \Gamma \Rightarrow \Delta, \varphi| H \quad G|\varphi, \psi, \Gamma \Rightarrow \Delta| H}{G|\psi, \Gamma \Rightarrow \Delta| H} \text { (Cut) }}{G|\Gamma \Rightarrow \Delta| H}
$$

Both cuts are performed on formulae of lower complexity hence eliminable by the induction hypothesis.

The cases involving the cut formulae $\square^{F} \varphi$ or $\square^{P} \varphi$ are a bit different since invertibility results are restricted. We must additionaly perform subsidiary induction on the height of the proof of the right premiss, similarly like in the proof of the basis. Fortunately the inductive step goes exactly like for atomic cut formula so it is not necessary to consider it again in full. The decisive case is with $\square^{F} \varphi$ principal in the right premiss; the situation looks like this:

$$
\frac{G\left|\Gamma \Rightarrow \Delta, \square^{F} \varphi\right| \Theta \Rightarrow \Lambda \mid H \quad \frac{G|\Gamma \Rightarrow \Delta| \square^{F} \varphi, \varphi, \Theta \Rightarrow \Lambda \mid H}{G\left|\square^{F} \varphi, \Gamma \Rightarrow \Delta\right| \Theta \Rightarrow \Lambda \mid H}\left(\square^{\mathrm{F}} \Rightarrow\right)}{G|\Gamma \Rightarrow \Delta| \Theta \Rightarrow \Lambda \mid H} \text { (Cut) }
$$

By inversion lemma on the left premiss we obtain: $G|\Gamma \Rightarrow \Delta| \Theta \Rightarrow$ $\Lambda, \varphi \mid H$ and $G|\Gamma \Rightarrow \Delta| \Theta \Rightarrow \Lambda, \square^{F} \varphi \mid H$. The latter, with the application of $(I W)$, yields the following: 


$$
\frac{G|\Gamma \Rightarrow \Delta| \varphi, \Theta \Rightarrow \Lambda, \square^{F} \varphi|H \quad G| \Gamma \Rightarrow \Delta\left|\square^{F} \varphi, \varphi, \Theta \Rightarrow \Lambda\right| H}{G|\Gamma \Rightarrow \Delta| \varphi, \Theta \Rightarrow \Lambda \mid H} \text { (Cut) }
$$

which with the former gives:

$$
\frac{G|\Gamma \Rightarrow \Delta| \Theta \Rightarrow \Lambda, \varphi|H \quad G| \Gamma \Rightarrow \Delta|\varphi, \Theta \Rightarrow \Lambda| H}{G|\Gamma \Rightarrow \Delta| \Theta \Rightarrow \Lambda \mid H} \text { (Cut) }
$$

The first cut has the right premiss with lower height so is eliminable by the induction hypothesis of subsidiary induction. The second cut is eliminable by the induction hypothesis of the main induction on complexity of cut formula. In case of cut formula $\square^{P} \varphi$ the proof is similar.

\section{References}

[1] A. Avron, A Constructive Analysis of RM, Journal of Symbolic Logic 52 (1987), pp. 939-951.

[2] A. Avron, Using Hypersequents in Proof Systems for Non-Classical Logics, Annals of Mathematics and Artificial Intelligence 4 (1991), pp. 225-248.

[3] A. Avron, The Method of Hypersequents in the Proof Theory of Propositional Non-Classical Logics, [in:] W. Hodges et al. (eds.), Logic: From Foundations to Applications, Oxford Science Publication, Oxford, 1996, pp. 1-32.

[4] M. Baaz, A. Ciabattoni and C. G. Fermüller, Hypersequent Calculi for Gödel Logics - a Survey, Journal of Logic and Computation 13 (2003), pp. 1-27.

[5] K. Bednarska and A. Indrzejczak, Hypersequent Calculi for S5 - the Methods of Cut-elimination, Logic and Logical Philosophy 24/3 (2015), pp. $277-311$.

[6] A. Ciabattoni, N. Galatos and K. Terui, From axioms to analytic rules in nonclassical logics, LICS (2008), pp. 229-240.

[7] A. Indrzejczak, Cut-free Hypersequent Calculus for S4.3, Bulletin of the Section of Logic 41:1/2 (2012), pp. 89-104.

[8] A. Indrzejczak, Eliminability of Cut in Hypersequent Calculi for some Modal Logics of Linear Frames, Information Processing Letters 115/2 (2015), pp. $75-81$. 
[9] A. Indrzejczak, Linear Time in Hypersequent Framework, The Bulletin of Symbolic Logic 22/1 (2016), pp. 121-144.

[10] O. Lahav, From Frame Properties to Hypersequent Rules in Modal Logics, LICS 2013.

[11] B. Lellmann, Axioms vs hypersequent rules with context restrictions, [in:] Proceedings of IJCAR, Springer 2014, pp. 307-321.

[12] B. Lellmann, Linear Nested Sequents, 2-Sequents and Hypersequents, [in:] TABLEAUX, Springer 2015, pp. 135-150.

[13] H. Kurokawa, Hypersequent Calculi for Modal Logics Extending S4, [in:] New Frontiers in Artificial Intelligence (2013), pp. 51-68, Springer, 2014.

[14] G. Metcalfe, N. Olivetti and D. Gabbay, Proof Theory for Fuzzy Logics, Springer 2008.

[15] F. Poggiolesi, A Cut-free Simple Sequent Calculus for Modal Logic S5, Review of Symbolic Logic 1 (2008), pp. 3-15.

[16] F. Poggiolesi, Gentzen Calculi for Modal Propositional Logics, Springer, 2011.

[17] G. Pottinger, Uniform Cut-free formulations of T, S4 and S5 (abstract), Journal of Symbolic Logic 48 (1983), p. 900.

[18] K. Schütte, Proof Theory, Springer, 1977.

Department of Logic

University of Łódź

Lindleya 3/5, Łódź

Poland

e-mail: andrzej.indrzejczak@filozof.uni.lodz.pl 